\title{
Annealing effects of polymers and their underlying molecular mechanisms*
}

\author{
G. S. Y. Yeh \\ University of Michigan, Ann Arbor, Michigan 98104, USA
}

\author{
R. Hosemann, J. Loboda-Čačković and H. Čačković \\ Fritz-Haber-Institut der Max-Planck-Gesel/schaft, Berlin, West Germany \\ (Received 3 July 1975)
}

\begin{abstract}
This paper indicates that changes in chain mobility, heat capacity, WAXS crystallinity, SAXS long period, SAXS peak intensity, specific volume and morphology as a function of increasing temperature, occur in three fairly distinct annealing ranges (I, II and III) that are more or less the same for all crystallized polymers with a lamellar morphology. It is shown that none of the proposed molecular models to date, including the well-known fold surface premelting model, can satisfactorily account for all the experimental data. However, a new molecular interpretation, based primarily on electron microscopy and SAXS studies of changes such as lateral 'melting' from edges of microparacrystallites $(m P C)$ within the lamellae seen at the annealing temperatures can account for the data. With our new molecular interpretation, the effect of temperature increase is established to result in a slight breakup of the laterally aligned $M P C$ within the lamellae at low annealing temperatures in range 1 , and selective lateral 'melting' of the exposed $m P C$ and recrystallization at higher annealing temperatures in ranges II and III, with the recrystallization being very limited in range III. Annealing effects seen in cold- or hot-drawn polymers with a fibrillar morphology can also be readily accounted for by this very general molecular mechanism occurring in the same annealing temperature ranges.
\end{abstract}

\section{INTRODUCTION}

The effects of annealing a semi-crystalline polymer with a lamellar morphology to near its melting point are fairly well known ${ }^{1-3,6}$. Substantial changes can be detected in molecular mobility, morphology, physical and mechanical properties. These changes are not only important from a user's point of view, but also in determining optimum conditions under which solid state processing techniques can be applied to fabrication of polymer products. However, in spite of the numerous extensive studies on this very important and practical subject of annealing in the past 10-20 years, we are still not very clear about the underlying molecular mechanisms responsible for the various observed annealing effects. There have been several proposed molecular mechanisms, all apparently very different from one another ${ }^{2}$, none of which is seen to be more satisfying in the explanation of annealing data than the other although all of them appear to have some degree of experimental justification.

One of the major difficulties in coming up with a better understanding of the annealing phenomena, is the dependency of observed changes on numerous factors such as annealing temperature, time and rate, prior thermal history and the physical state (drawn vs. undrawn or single crystal vs. bulk) of the sample, as well as its chemical structure and composition (molecular weight, degree of branching, etc.). These factors often contribute to a shifting and/or narrowing of the temperature ranges in which the changes may occur, to the degree of reversibility and to the magnitude of the observed changes which may lead to the various proposed molecular mechanisms. Another factor, which is less important but contributes to some degree of confusion,

\footnotetext{
* Dedicated to Professor G. Manecke on the occasion of his 60 th birthday
}

lies in the choice of words in describing some of the changes occurring well below the melting point. For example, it was cautioned by Hoffman ${ }^{4}$ several years ago, that the word 'premelting' should be used with care in describing certain annealing phenomena allegedly associated with melting in polymers.

It is the purpose of this paper to show and discuss briefly, that most of the annealing changes occur in three more or less distinct temperature ranges, to examine more critically the three most popular proposed molecular mechanisms, to introduce and discuss the validity of some other annealing mechanisms based on direct morphological changes observed at the annealing temperature, and to see how they can be applied to the understanding of annealing phenomena which occur in cold or hot drawn systems.

Whenever possible, we shall discuss primarily the annealing changes measured on as-crystallized, well-equilibrated lamellar systems kept at the annealing temperatures. Discussions on measurements made on samples after quenching will be limited and pointed out in order to minimize sources of confusion. For the sake of clarity and brevity we shall restrict our present discussions to annealing phenomena which occur upon temperature increase. The many interesting effects which occur upon temperature decrease after annealing (at higher temperatures) will be discussed only for clarifying points raised.

\section{SEPARATION OF ANNEALING CHANGES INTO THREE ANNEALING TEMPERATURE RANGES}

As the annealing changes are very much dependent on a number of factors as pointed out in the introduction, it is difficult to make any precise separations of the changes 


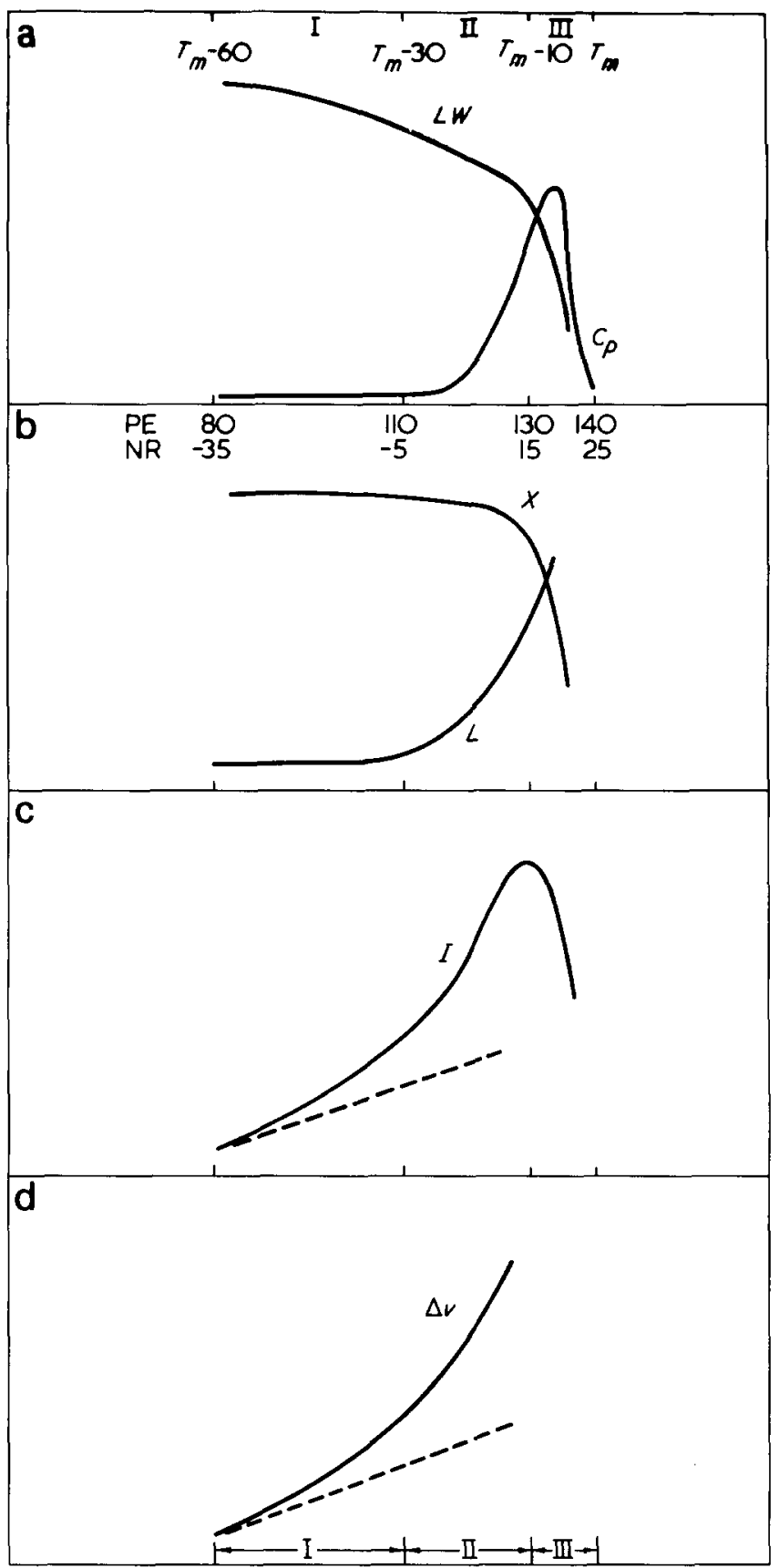

Figure 1 (a)-(d) are schematic drawings showing some well-known annealing effects in crystallized polymers upon heating to tempera. ture ranges I, II and III. (a) n.m.r. line-width, $L W$ (inversely proportional to molecular mobility) of the crystalline polymer component and specific heat, $c_{p}$; (b) WAXS crystallinity, $x$, and SAXS long period, $L ;$ (c) SAXS intensity, $/$; (d) specific volume difference, $\Delta V$

with annealing temperature. However, after examining numerous annealing data, we have come to the conclusion that there are certain distinct characteristics e.g. the magnitude of changes upon temperature increases or the degree of reversibility of changes upon temperature decrease, which allow us to assign the changes according to three annealing temperature ranges I, II and III.

Figures $1 a-1 d$ represent schematically the generally reported changes in chain mobility from n.m.r. linewidth $(L W)$, specific heat $\left(C_{p}\right)$, WAXS crystallinity $(X)$, SAXS long period $(L)$, SAXS intensity $(I)$, and difference in specific volume $(\Delta V)$, and the three approximate temperature ranges in which the changes are observed to vary for most polymers when heated to near their melting points. The lines (dotted) in Figures $1 c$ and $1 d$ are inserted to show the expected changes in SAXS intensity and specific volume, assuming no changes in the relative respective amounts of the two crystalline and non-crystalline components of the polymers, (see references 2 and 3 ).

The three approximate temperature ranges are: (I), from $\sim\left(T_{m}-60\right)$ to $\sim\left(T_{m}-30\right)$ (or to a temperature near the original crystallization temperature or to the first annealing temperature of a crystallized polymer); (II) from $\sim\left(T_{m}-30\right)$ to $\sim\left(T_{m}-10\right)$; and (III) from $\sim\left(T_{m}-10\right)$ to $\sim T_{m}$ (the melting point). They are generally believed to be more or less the dividing temperature ranges in which the above mentioned annealing effects are characteristically seen to be changing in nearly all well-equilibrated, crystallized polymers with a lamellar morphology. For example, the range will be from $\sim 80^{\circ}$ to $110^{\circ} \mathrm{C}, \sim 110^{\circ}$ to $130^{\circ} \mathrm{C}$ and $\sim 130^{\circ}$ to $140^{\circ} \mathrm{C}$ for polyethylene crystallized by slow cooling, and from $\sim-35^{\circ}$ to $-5^{\circ} \mathrm{C}, \sim-5^{\circ}$ to $15^{\circ} \mathrm{C}$ and $\sim 15^{\circ}$ to $25^{\circ} \mathrm{C}$ for natural rubber also when crystallized by slow cooling. The melting points are assumed to be $140^{\circ} \mathrm{C}$ and $25^{\circ} \mathrm{C}$ respectively for polyethylene and natural rubber crystallized under isotropic conditions.

In the annealing temperature range $I$ as shown in Figures $1 a-1 d$, practically no changes are observed in $C_{p}, X$ or $L$ while some small increases are already noticeable in degree of chain mobility as reflected in the decrease in $L W$ from n.m.r. and in $\Delta V$; the specific volume increase ${ }^{1}$ indicating an apparent decrease in volume crystallinity. There is also a noticeable increase in SAXS intensity over and above the expected increase due to the thermal expansions of the respective crystalline and amorphous components ${ }^{1}$.

All the annealing effects including the morphological changes (to be discussed later) detected in range I, are generally found to be small and generally reversible with temperature, i.e. upon temperature decrease to the original starting temperature the changes disappear. This is quite different from the annealing phenomena seen in annealing temperature ranges II and III, where the degree of reversibility appears to be generally limited.

In annealing range II the higher temperatures generally bring about greater increases in the changes $\left(L W, I\right.$ and $\left.V_{\text {sp }}\right)$ started in range $I$. In addition, other very noticeable changes begin to show up in this temperature range, i.e. $C_{p}$ and $L$ (see Figures $l a$ and $l b$ ). The crystallinity $X$ as determined from WAXS, however, remains practically unaffected in this temperature range, after long annealing times. The effect of annealing time in this temperature range on $X$ was first reported by Fischer and Schmidt on bulk and single crystals of polyethylene ${ }^{5}$. Their data showing a loss of WAXS peak intensities at early stages of annealing, indicated some type of melting, followed by some type of recrystallization, which resulted in the recovery of the WAXS peak intensities.

Similar interpretation of annealing time effects, noted above for WAXS peak intensity changes, has also often been made for density changes detected in bulk and single crystal samples of polyethylene after quenching to room temperature from annealing temperatures in range II. However, the interpretation of premelting and surface melting and recrystallization based on such density data alone, is less convincing than the $X$-ray results.

In annealing range III whatever partial (but real) melting process has started in range II, continues into the higher annealing temperature range. However, the 'recrystallization process', cannot be extended to too high a temperature because of the limited supercoolings (and therefore 
smaller nucleation rate) at higher temperatures. Consequently, one can see in this temperature range, that there is a substantial change in molecular mobility as judged by the decrease seen in $L W$, increases in $\Delta V$ and $L$ (due to recrystallization at smaller supercoolings), and decreases in WAXS crystallinity as well as SAXS intensity.

\section{PRESENT STATUS CONCERNING UNDERLYING MOLECULAR MECHANISMS}

The three most often cited molecular mechanisms to account for the annealing effects, in particular the lamellar thickness increase, are: A, refolding by 'solid state' diffusion; B, 'selective melting of thinner lamellae and recrystallization to thicker lamellae' and $\mathrm{C}$, the fold surface 'premelting'. Their merits have been reviewed extensively by Fischer ${ }^{2}$ and therefore will not be belaboured further here. Nevertheless, it is felt that a brief discussion of these three molecular models, in light of all the annealing effects cited above, is appropriate for any new concepts of annealing mechanisms to be introduced later.

\section{'Solid state' diffusion}

Refolding by a 'solid state' snaking type long chain diffusion process along the $c$-axis, was based mainly on the observation that the $c$-axis in thickened portions of polyethylene single crystals after annealing to $125^{\circ} \mathrm{C}$, remains perpendicular to the lamellar surface when examined at room temperature ${ }^{6}$. As was originally proposed, this seemed a very probable process especially in the higher annealing temperature ranges, where molecular mobility may be sufficiently high and lattice sufficiently expanded, for such gliding movements. However, in light of the annealing time effects discussed earlier concerning WAXS intensity changes in annealing temperature range II for polyethylene single crystals or bulk polyethylene and the detectable changes in annealed morphology (to be discussed later), one questions the general validity and applicability, if one defines solid state diffusion as diffusion of atoms or molecular chain segments in a lattice which retains its 3 -dimensional crystalline property. Some limited refolding by solid state diffusion, of course, can and probably does occur, e.g. in non-equilibrated systems, such as cold or hot drawn systems containing numerous highly stressed defective crystals. However, in such systems we may be dealing with numerous 'crystalline' regions, that can hardly be considered as solid to begin with and annealing of such systems even at temperatures below those in ranges II and III may lead to limited growth of crystallites by a pseudo-recrystallization process.

\section{Selective melting of thinner lamellae}

Since the lamellae in crystallized polymers generally consist of a distribution of thicknesses, the proposal that thinner lamellae melt first (and therefore selectively), within a stack of lamellae appears to be a reasonable one and is still considered by many, including Hoffman and Kilian ${ }^{4}$, together with some degree of recrystallization, to be the most reasonable explanation for the observed long period increase. Fischer's objection to this model centres entirely around the disagreement between experimental SAXS peak intensity measurements (see Figure $1 c$ ) and theoretically predicted values which should show a decrease rather than the observed increase, if melting of lamellae occurs selectively in a stack of lamellae that can scatter X-ray coherently. Al- though this is a reasonable objection which will be amplified further later there is some indication that a modified form of selective melting, also to be discussed in later, may indeed be occurring in annealing temperature range III.

\section{Fold surface 'premelting'}

This model indicates that starting at some temperature, the fold surfaces 'premelt' gradually inwards towards the crystalline core along the chain axis, until the whole lamellar crystal is melted at the $T_{m}$. This premelting model appears to be able to account for nearly all the experimental evidences of annealing effects, in particular the evidence concerning the SAXS integrated and peak intensity increase with annealing temperatures as has been found in a number of polymeric systems ${ }^{5}$. However, even if it is a correct model, as was pointed out earlier by Luch and $\mathrm{Yeh}^{9}$, it can be applied to lamellar systems with only very little increase in lamellar thickness upon annealing, since the melting of fold surface does not directly lead to large increases in amorphous thicknesses, as have been observed in a number of cases in annealing temperature ranges II and III. Furthermore, for the model to apply, the polymers must have initial crystallinities of greater than $50 \%$; otherwise, a predicted decrease in SAXS peak or integrated intensity will result. However, Luch and Yeh ${ }^{7}$ studied natural rubber which has a maximum crystallinity of well below $50 \%$ and showed the same kind of SAXS peak intensity changes (Figure lc) with an initial increase followed by a decrease at annealing temperatures close to $T_{m}$.

It should be mentioned that the difficulty with the surface premelting model to explain large changes in lamellar thicknesses (in regions II and III), was not encountered when the model was originally conceived and applied ${ }^{8}$ to explain the SAXS intensity changes for polyethylene single crystals in annealing temperature range I where there is hardly any change in long period (Figure $1 b$ ). Therefore, it will be of interest in this respect to re-examine the experimental evidence that has led to the surface premelting model. The evidence is in the SAXS intensity change (Figure $1 c$ ). Nukushina, Itoh and Fischer ${ }^{8}$ using PE single crystal mats and Schultz, Robinson and Pound ${ }^{9}$ using bulk PE have noted reversible peak intensity changes up to four-fold with hardly any changes in long period $L$. Since this intensity increase was found to be much greater than could be accounted for by density changes owing to thermal coefficients of expansion of the two respective phases, (see lines (dotted) in Figures $l c$ and $l d$ ) it was, albeit incorrectly, suggested that the increase must be due to increase in the amorphous thickness (or in the decrease of volume fraction of crystals) and, therefore, melting from the lamellar fold surface inward at the expense of the crystalline core.

\section{MOLECULAR MECHANISM(S) BASED ON MORPHOLO- GICAL OBSERVATIONS}

The above discussions, although brief, clearly indicate a degree of inadequacy existing in all the three proposed models, including surface 'premelting', to account for the various phenomena that appeared during annealing.

In addition to the shortcomings mentioned earlier, the premelting and the surface melting models, seldom discuss specifically whether and how a recrystallization process follows the 'melting' process in either range II or III. If a recrystallization process takes place, i.e. one to increase the crystal thickness of the remaining, unmelted lamellae 
(or the unmelted crystalline cores in the case of Fischer's fold surface premelting model), one wonders how this crystal thickness increase comes about without greatly disturbing the crystalline lattice of the remaining, unmelted lamellae in a stack of well-packed lamellae. Any substantial disturbance will lead to melting of the whole stack of lamellae. Otherwise, one has to suggest some kind of 'solid state' diffusion process by which the molecules from the melt regions can participate in the thickening process of the previously unmelted crystalline core of the lamellae.

It seems that on the one hand we need to propose some sort of melting, albeit of a limited extent, to explain most of the observed phenomena; while on the other hand we seem to need also, some sort of 'solid state' diffusion process to explain how the crystal thickness increase takes place. As will be shown below, these problems quickly disappear when one finds that the suggested 'premelting' in annealing temperature range $I$, and the suggested partial melting/recrystallization process and the 'solid state' diffusion process in annealing temperature ranges II and III, may all appear to have taken place. Even though the underlying molecular mechanism(s) which gives rise to the annealing changes seen in ranges I, II and III, may be quite different from the proposed models.

The underlying molecular mechanism(s) to be discussed below is primarily based on direct observations of morphological changes in polymers annealed at the temperature. The starting polymer has the well-known lamellar texture, which is typical of well-equilibrated, well-crystallized systems, in either the oriented or unoriented state. Another point to be made here is that the basic crystalline units in crystallized polymers are not the lamellae, as had been gradually assumed over the years, but rather the smaller microparacrystallites $(m P C)$ or nodules within the lamellae. This has been established independently by Hoseman $n^{10}$ using $\mathrm{X}$-ray scattering techniques and Yeh using direct morphological observations ${ }^{11}$. It is particularly useful to keep this fact in mind when one tries to evaluate the generality of the observations and the molecular mechanisms discussed below.

Direct morphological studies of unoriented polymers or single crystals after annealing in temperature range $I$ and examined at room temperature, do not generally reveal any detectable changes. This can be due to a number of reasons, e.g. the problem with detecting changes from unoriented specimens or single crystals when examined from vertical positions perpendicular to the lamellar surface, or with recovered morphological changes when examined at room temperature, or with the degree of resolution due to proper examining techniques and specimen preparations, etc.

In our earlier studies ${ }^{12,13}$ of strain-crystallized natural rubber (cis-polyisoprene) and gutta percha (trans-polyisoprene), we were able to detect definite changes in morphology at the annealing temperature in range $I$ by means of $\mathrm{OsO}_{4}$ staining technique and examining the edges of stacks of well-oriented lamellae in a direction perpendicular to the $c$-axis. An example of the changes is reproduced here in Figure $2 b$ for natural rubber when annealed to $-15^{\circ} \mathrm{C}$ (in the annealing temperature range $\mathrm{I}$ ) and $\mathrm{OsO}_{4}$ stained at the annealing temperature. It shows a slight, but noticeable 'breakup' of the well-formed lamellae originally present in the sample (Figure $2 a$ ) into blocks of about $100 \AA$ lateral size as deduced by the attained 'amorphous' boundaries in between the $m P C$. Figures $3 b$ and $3 c$ show schematic representations of the $m P C$ originally present within the lamellae and after their 'breakup'. We suggest that the 'amorphous' boundaries arise from an increase in localized intrachain defects at the $m P C$ grain boundaries upon reaching annealing temperature in range $I$. They could very well contribute to the $\alpha_{1}$ type of molecular motions as detected and assigned by Takayanagi to be occurring within the mosaic block grain boundaries of polyethy. lene single crystals ${ }^{14}$ in the same annealing temperature range I. Since intrachain defects involve a contraction of chain segments at the mosaic crystal grain boundaries, the contraction will create vacancies at the lamellar fold surface. They contribute to a greater lowering of the density in the interfacial region, inbetween the crystalline lamellae than that of the crystalline lamellar cores, thereby leading to a larger difference in density $\left(\rho_{c}-\rho_{a}\right.$ or $\left.\Delta \rho\right)$ between the two phases. Therefore over and above the values based on changes due only to thermal coefficients of expansion there exists an extra $\Delta V$ increase (Figure $1 d$ ). The SAXS intensity increase in this temperature range I (Figure 1c) is then the direct result of vacancies created at the fold surface boundary since intensity is proportional to $(\Delta \rho)^{2}$, somewhat similar to the origin of substantial SAXS intensity increase observed for $n$-paraffins upon annealing ${ }^{15}$.

This type of morphological change was observed to be reversible with temperature, i.e. upon cooling the lamellae were found to be coherent again, as long as the annealing temperature stays inside range I, which also explains why the changes in SAXS intensity and specific volume are also found to be reversible with temperature. Therefore, it is not necessary to have a surface 'premelting' type of molecular mechanism to explain either the SAXS intensity increase or its reversibility with temperature. Furthermore, one would not expect this kind of intrachain defect to cause any significant change in molecular mobility, specific heat, crystallinity or long period, as, indeed, is generally found to be the case (Figures $1 a$ and $1 b$ ).

Upon entering annealing temperature range II, an entirely different morphology arises. First, there is clear indication from direct observations ${ }^{12,13}$ that whole or parts of $m P C$ or nodular crystallites melt within the lamellae. This is again deduced from careful examinations of stacks of well-oriented lamellae of natural rubber and trans-poly. isoprene annealed and $\mathrm{OsO}_{4}$ stained at the annealing temperatures. When held at the annealing temperatures for a sufficient length of time, the true melting process within the lamellae is followed by a recrystallization process, resulting in more stable, thicker (along the $c$-axis) $m P C$ which then repack and realign with one another to form apparently smooth and filled* lamellae, at long annealing times. The lamellae now have thicker crystalline as well as thicker amorphous regions, as for example, shown in Figure 4 for trans-polyisoprene. The relatively constant WAXS crystallinity (Figure $1 b$ ) can now be explained by nearly the same percentage increase of the crystal $\left(l_{c}\right)$ and the amorphous $\left(l_{a}\right)$ thicknesses. In an ideal two phase lamellar structure, the WAXS crystallinity should be the same as the linear crystallinity $l_{c} /\left(l_{a}+l_{c}\right)$ directly measured from the electron micrographs, if the lamellae are present throughout the volume as recently demonstrated by $\mathrm{Yeh}^{16}$. The true melting process (which continues in range III) also causes substantial molecular motion and increase in specific heat (Figure 1a), while the increase in SAXS long period is due

\footnotetext{
* Polyethylene single crystals when annealed to this temperature range, often showed holes within single lamellae after a nnealing and cooling to room temperature ${ }^{6}$. This is due to unavailability of neighbouring erystallites, above or below the lamella, for space filling.
} 

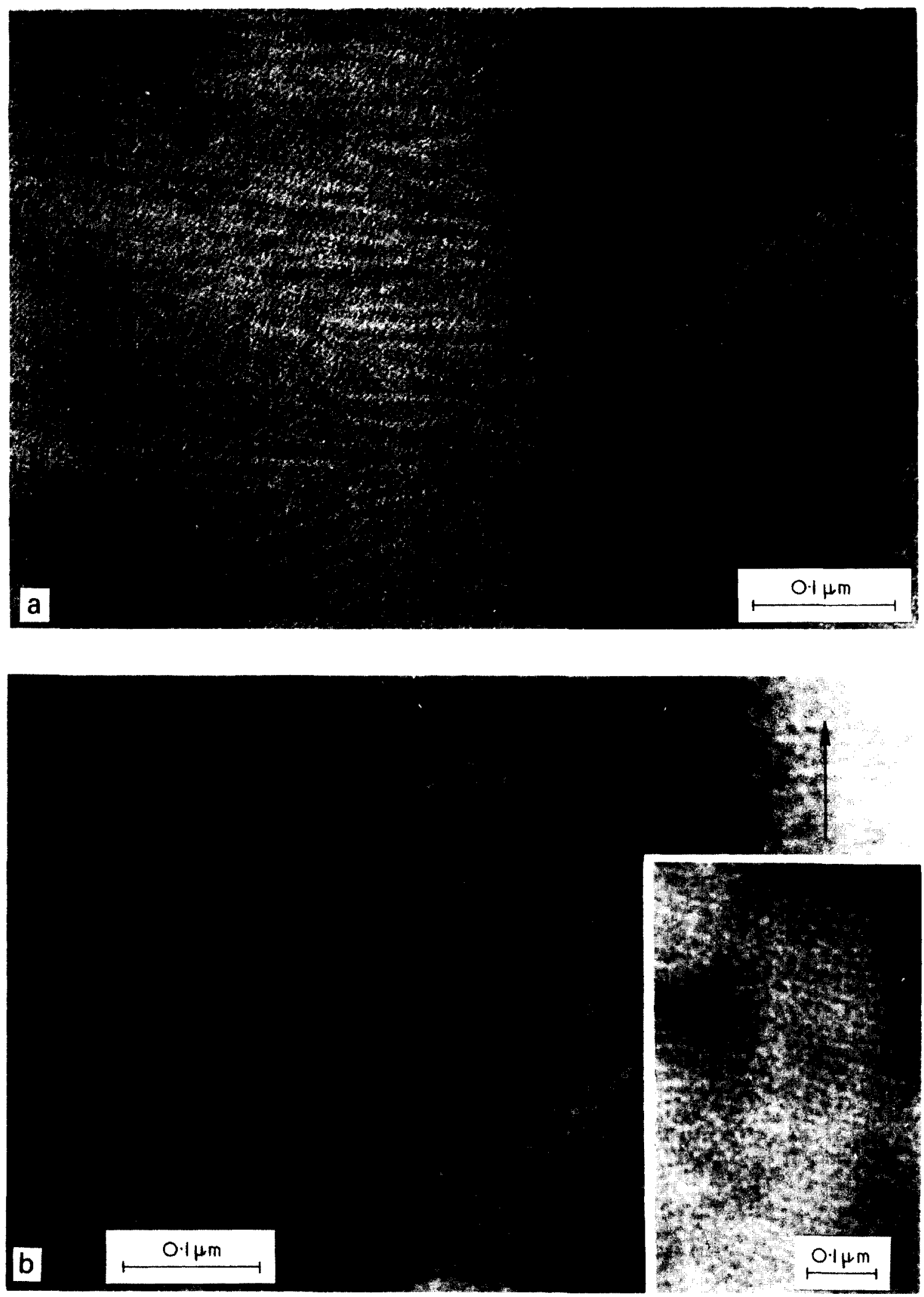

Figure 2 (a) Typical bundles of 'lamellae', seen edgewise in strain-crystallized natural rubber after $\mathrm{OsO}_{4}$ staining at $-28^{\circ} \mathrm{C}^{12}$; (b) Individual $m P C$ seen with in lamellae after annealing and $\mathrm{OsO}_{4}$ staining at $-15^{\circ} \mathrm{C}$ in range $\mathrm{I}^{12}$

to an increase in both $l_{a}$ and $l_{c}$ (Figure 4). If the melting involves melting of smaller or thinner $M P C$ within the lamellae, as we believe it does, then a slightly modified selective $m P C$, rather than lamellar, melting model will apply very well here. This will be shown later. Nevertheless, one can safely eliminate the fold surface 'premelting' model involving longitudinal melting from the lamellar fold surface inward, or the selective melting of the thinner lamellae within a stack of lamellae, since neither are observed ${ }^{12,13}$ The basis for the objection to the fold surface premelting model in annealing temperature ranges II (and III) is therefore quite different from that in range I, where no substantial 'premelting' of the lamellae (either laterally or longitudinally) is observed. 

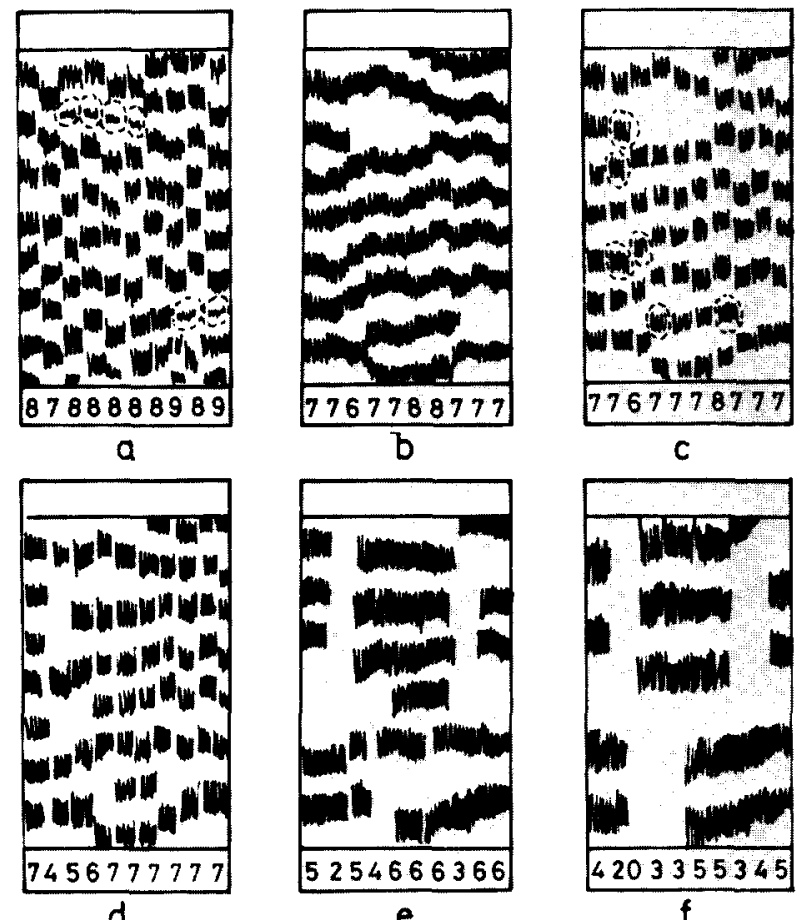

Figure 3 Structure of the $m P C$ in the three temperature ranges. Schematically 10 columns of $m P C$ extend over the whole picture. The numbers on the bottom give the number of $M P C$ along the vertical direction. (a) Below range I in non-equilibrated drawn systems; the $m P C$ are nematic-like; some of them can be very thin (circled). (b) Beginning of range I. The $M P C$ are laterally aligned to form a smectic-like layer structure (originally present in wellequilibrated lamellae systems). Thin $m P C$ are partially melted upon reaching range $I$ in non-equilibrated drawn systems. (c) In range I the chain segments in lateral boundaries of $M P C$ have higher mobilities, will begin to melt and contract to produce vacancies at the fold surface and unprotected lateral boundaries (circled). (d) In range II lateral melting of $m P C$ may start at the unprotected lateral boundaries with simultaneous recrystallization to thicker $m P C$, resulting in the increase of long period. (e) At end of range II, after long annealing times, most of the melted regions have recrystallized epitaxially onto the more stable thicker $m P C$, which are now realigned to form smoother lamellae. (Only a few regions may still remain molten). (f) In range III, only thicker $M P C$ can resist the melting process, while many other regions remain molten. The volume fraction $\gamma$ of bundles and hence $l$, the crystallinity $X$ and n.m.r. line-width $L W$ decrease drastically; whereas the long period $L$ may still keep on increasing

In annealing range III, the partial melting process of $m P C$ which started in region II occurs with a much greater intensity at higher temperatures. However, the recrystallization process cannot be expected to extend to too high a temperature, since nucleation rate is a strong function of supercooling. Consequently, some of the melted regions remain selectively and permanently molten, contributing to substantial increases in molecular mobility (as judged by a substantial decrease in $L W$ ) and specific volume, and decreases in WAXS crystallinity, while other molten regions may still recrystallize into lamellae of greater thicknesses and therefore larger SAXS long periods consisting of substantially greater crystalline and amorphous thicknesses (Figure 4). An example is given in Figure 5 showing the presence of permanently molten regions as well as shorter segments of lamellae. A similar possibility of permanent melting and thickening of portions of lamellae, is seen from recrystallized morphology of polyethylene single crystals, observed at room temperature after annealing to higher temperatures in region III $^{1}$. The deduction of permanent melting in polyethylene single crystals occurring at the annealing temperature was based on the observed disoriented fibrillar crystallites present within the lamellae at room temperature.

The permanently melted regions directly detected in Figure 5 can also explain the much lower volume crystallinity measured at annealing temperatures in region III, as reported earlier by Fatou and Mandelkem ${ }^{17}$ for polyethylene of various molecular weights.

\section{Melting and recrystallization process}

We believe that the melting starts at the grain boundaries between the $M P C$ where the intrachain defects have become substantially increased upon heating to annealing temperature range I (Figure 3c). Because of their small size $\left(\sim 100 \times 100 \times 100 \AA^{3}\right)$, the much weakened exposed boundaries can be sources of instability and initial melting (Figure $3 d$ ). Eventually the melted region can recrystallize epitaxially onto the more stable, thicker $M P C$ which had remained unmelted, with a greater lamellar thickness corresponding to a particular smaller supercooling (Figure $3 e$ ). The new crystals will have the same $c$-axis orientation as the old crystals upon which the new crystallites have nucleated. One can easily show that the melting and recrystallization can lead to a substantial reduction in total surface free energy. For example, an increase in lamellar thickness to $120 \AA$ from a $100 \AA$ thick cube, will result in a reduction in total surface free energy of about $12 \%$ owing to reduction of high energy fold surface area from $100 \times 100 \AA^{2}$ to about $91 \times 91 \AA$, assuming a reported ${ }^{18}$ end fold surface

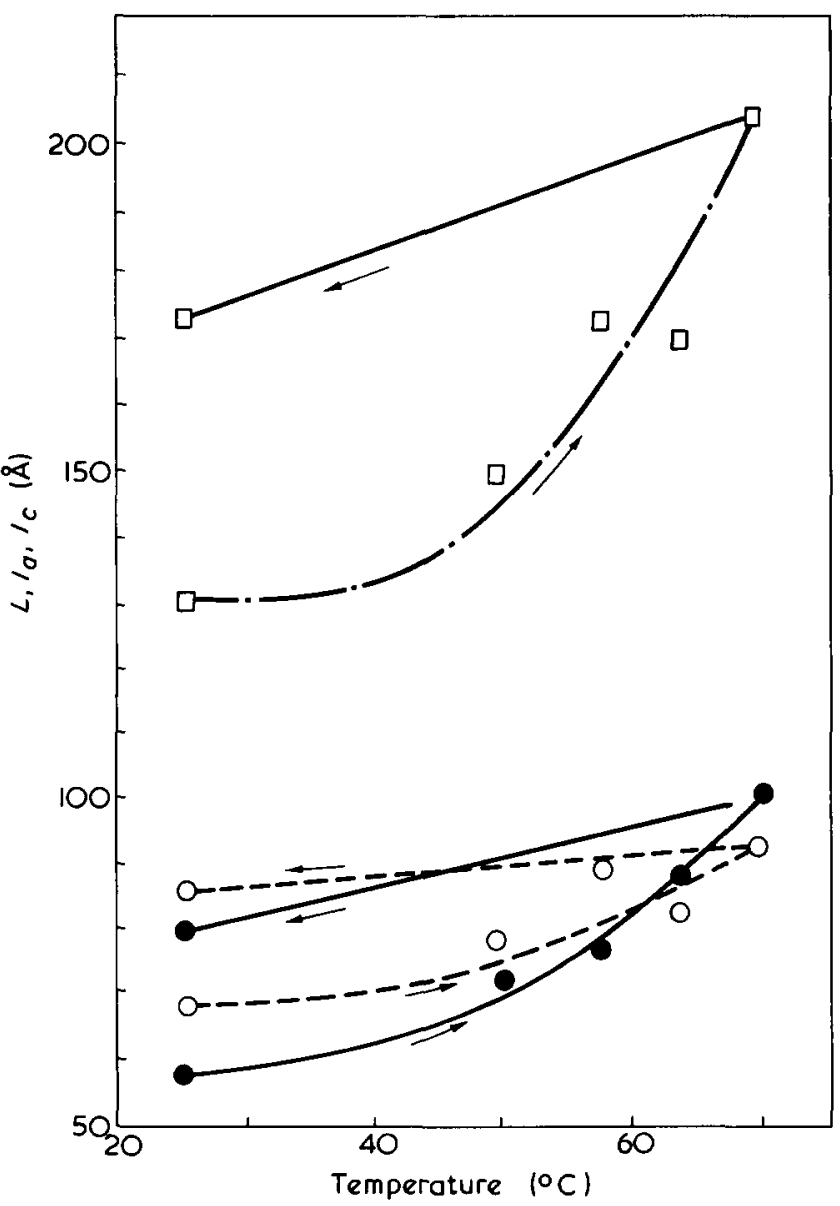

Figure 4 E.m. long period, $L$, plotted against annealing temperature for a strain-crystallized trans-poly isoprene ${ }^{13}(-\cdot-\cdot-\cdot)$. Variation in crystal thickness, $I_{c},(--)$ and variation in amorphous thickness, $I_{a}(-)$ 


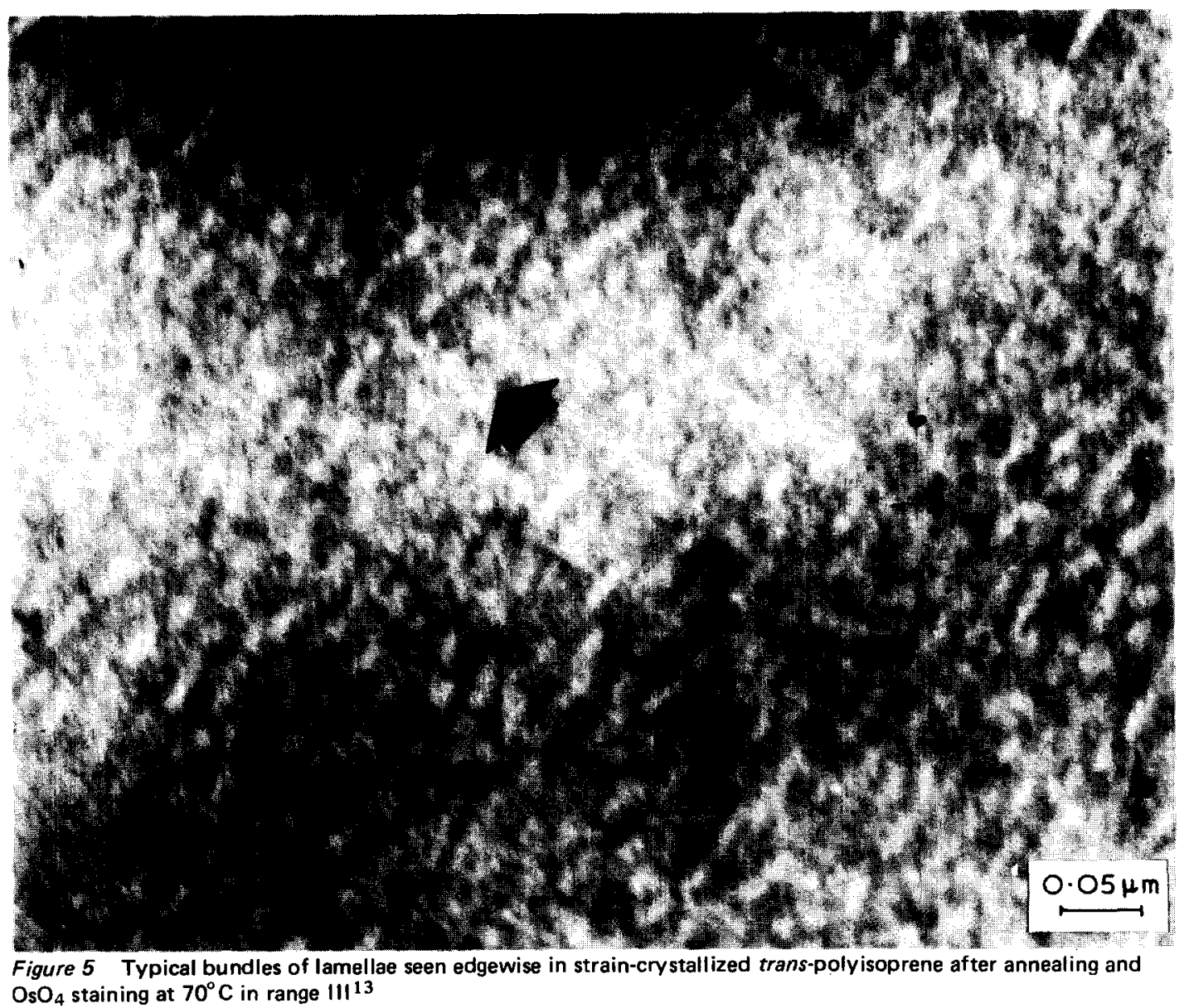

free energy of about $80 \mathrm{ergs} / \mathrm{cm}^{2}$ and a reasonable lateral side surface free energy of about $10 \mathrm{ergs} / \mathrm{cm}^{2}$. The new $m P C$ can further reduce their total surface free energy through lateral alignment to form new lamellae consisting of some old and some new $M P C$.

Figures $3 c$ and $3 d$ schematically give an example of this melting and recrystallization process. Along columns 2, 3, 4 and 7 six $m P C$ with unprotected lateral surfaces show up (circled) in Figure $3 c$ upon annealing to range I. (Prior to reaching temperature I, the lamellae have the smooth fold surface as shown in Figure $3 b$.) Upon reaching temperature range II (Figure $3 d$ ), the $m P C$ (circled) become molten in either column $2,3,4$ or 7 . Therefore the number of long periods in these columns has decreased in range II; column 3,4 or 7 by one and column 2 by 3 , from 7 to 4 . The average number of long periods in the 10 columns has diminished from $70 / 10$ or 7.0 in Figure $3 c$ to $64 / 10$ or 6.4 in Figure $3 d$. The average long period is therefore $9 \%$ larger in Figure $3 d$ than in Figure $3 c$. Lateral alignment of the new and old $m P C$ in further reduction of total surface free energy, is shown in Figures $3 e$ and $3 f$.

With the type of lateral melting of $m P C$ similar to the lateral melting proposed by Hosemann in $1962^{19}$, recrystallization and repacking, one can readily explain how the formation of new lamellae comes about, without resorting to nearly complete melting, as well as all the other annealing effects observed in annealing ranges II and III, including SAXS intensity and substantial long period increases, while the X-ray crystallinity remains relatively constant at long annealing times (Figure 1). Unlike the cause of SAXS intensity change in annealing range I which is due to greater differences of densities caused mainly by the vacancies introduced at the fold surface, the increase in SAXS peak intensity in annealing temperature ranges II and III, is caused primarily by increases of the thickness of lamellae (due to increases in $l_{a}$ and $l_{c}$ ) which is not discussed in the various idealized two-phase lamellar models in an earlier section. This process continues into annealing temperature range III. However, because of the limited driving force at higher temperature (smaller supercooling) substantial permanent melting will set in and lead to a reduction of the number of bundles (Figure 5), resulting in a substantial loss of crystallinity $X$ and in the observed drop of SAXS intensity (Figures $l b$ and $1 c$ ). The following formulae give the quantitative expressions.

\section{Peak intensity of SAXS}

The formulae of SAXS for an idealized lamellar twophase model, do not take into account the lateral boundaries existing between the adjacent $m P C$ within a given lamella. Therefore they cannot adequately describe the changes in peak intensity $I_{p}$ for many of the observed structures that may differ appreciably from an ideal lamellar two-phase model. However, it can be shown (see below), that with a slight modification of the basic formulae from the paracrystalline theory ${ }^{19}$ the changes in peak intensity for an oriented polymer can be readily accounted for by the changes in structure as a function of temperature. For example it has been demonstrated that a two-dimensional analysis of the SAXS of oriented samples can also give information about the shape of the $m P C$ and their mutual positions ${ }^{20-22}$, but this generally requires the use of a pinhole-camera. But even with the use of a camera with a slit orthogonal to the fibre axis some information can still be obtained of the two phases whether or not unbounded lamellae exist.

Let $f(b)$ be the scattering amplitude of one $m P C$ and $Z(b)$ the macroparacrystalline (MPC) lattice factor of an 
unbounded assembly of $m P C$ (if they are all pointlike). If $\left|S^{2}(b)\right|$ is the shape factor of the volume of a bounded assembly of $m P C$, the SAXS is given by:

$$
I(b)=n\left[\bar{f}^{2}(b)-\bar{f}^{2}(b)\right]+\frac{1}{v} \bar{f}^{2}(b) \overparen{Z(b)\left|S^{2}(b)\right|}
$$

$n$ is the number of $m P C$ in the assembly, which occupies the volume $V$ and $v=V / n$ that of one $m P C$.

In the case of polymers having a lamellar structure there exist $M$ bundles, each containing $N$ lamellae in the average. If $b_{3}$ is the axis of one of these bundles in the reciprocal space, then $Z_{3}(b)$ is the intensity function of the centres of lamellae of an unbounded bundle:

$$
Z_{3}(b)=R \exp \frac{1+F_{3}(b)}{1-F_{3}(b)} ; F_{3}(b)=\mathfrak{H}_{H_{3}}(x)
$$

$H_{3}(x)$ is the frequency distribution of a vector $x$ between the centres of gravity of adjacent lamellae and $\mathbb{1}=$ $\left[\int \exp -(2 \pi i b x) \mathrm{d} x\right]$ the symbol of Fourier-transform. The vector $x$ expands the physical and $b$ the reciprocal space. If $l_{c}$ is the mean thickness of the lamellar crystalline cores and $l_{a}$ that of the amorphous interlayer, the intensity of one bundle along the $b_{3}$-axis of the reciprocal $b$-space is proportional to equation (1) with:

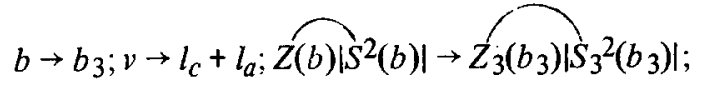

$$
\begin{aligned}
& \mathrm{f}(b) \rightarrow \mathrm{f}_{3}\left(b_{3}\right)=\frac{\sin \pi l_{c} b_{3}}{\pi b_{3}} .
\end{aligned}
$$

$f_{3}\left(b_{3}\right)$ is proportional to the scattering amplitude of one lamellae which consists of many $m P C$ packed next to each other in the lateral direction. The symbol $\cap$ stands for a convolution product. In equation (1) there is a threedimensional convolution product of the three-dimensionally defined function $Z$ and $\left|S^{2}\right|$; in equation (3) there is a onedimensional convolution product of the one-dimensionally defined function $Z_{3}$ and $\left|S_{3}{ }^{2}\right|$.

If all the lamellae are cylindrical discs with a diameter $D$ and if $\Delta \rho$ is the electron density difference between the 'crystalline' and the 'amorphous' phase of a lamella one obtains for the integral intensity of the SAXS along a line, at a constant distance $b_{3}$, parallel to the equator:

$$
\begin{aligned}
I\left(b_{3}\right)= & \iint I\left(b_{1}, b_{2}, b_{3}\right) \mathrm{d} b_{1} \mathrm{~d} b_{2}=(\Delta \rho)^{2} \frac{\pi D^{2}}{4}\left(\frac{\sin \pi l_{c} b_{3}}{\pi b_{3}}\right)^{2} \times \\
& \frac{1}{l_{c}+l_{a}} Z_{3}\left|S_{3}{ }^{2}\right|
\end{aligned}
$$

For convenience the first term of equation (1) is neglected here, because it does not affect the final results very much $^{21}$ The application of the paracrystalline theory to the macroparacrystalline lattice of the lamellae will now be introduced. (It should be pointed out here that the exact onedimensional solution of Hermans ${ }^{23}$, often used by Tsvankin et al. $^{24}$ in avoiding statistical overlapping of adjacent lamellae cannot be generalized to a three-dimensional bundle of lamellae ${ }^{25}$.)

Equation (2) shows that $Z_{3}$ has its first peak at $b_{3}=$ $1 /\left(l_{a}+l_{c}\right)$ with a maximum value:

$$
Z_{3 \max }=\frac{1+\left|F_{3}\left(b_{3 m}\right)\right|}{1-\left|F_{3}\left(b_{3 m}\right)\right|} ; b_{3 m}=\left(l_{c}+l_{a}\right)^{-1}
$$

$Z_{3}$ has an integral width $\delta b_{Z}$ given by ${ }^{25}$ :

$$
\delta b_{Z}=\frac{1}{2\left(l_{c}+l_{a}\right)}\left(1-\left|F_{3}\left(b_{3 m}\right)\right|\right)
$$

$\left|S_{3}{ }^{2}\right|$ on the other hand has an integral width given by:

$$
\delta b_{s}=\frac{1}{\left(l_{a}+l_{c}\right) N}
$$

The peak intensity depends on the relative contributions of these two quantities and has extreme values as follows:

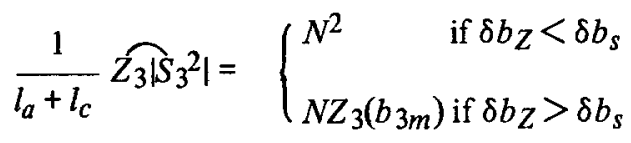

Since the modulus of $F_{3}(b)$ in equation (5) can be approximated as:

$$
\begin{aligned}
& \qquad\left|F_{3}\left(b_{3}\right)\right| \sim \exp \left(-2 \pi^{2} g^{2} h^{2}\right) \\
& \text { where } g^{2}=\frac{\frac{\left(l_{c}+l_{a}\right)^{2}}{\left(l_{c}+l_{a}\right)^{2}}-1 ; h=b_{3} /\left(l_{c}+l_{a}\right)}{}
\end{aligned}
$$

introducing this into equation (6) one obtains for the integral width of the first peak at $h=1$ of $Z_{3}$ :

$$
\delta b_{Z}=\pi^{2} g^{2} /\left(l_{a}+l_{c}\right)
$$

$g$ generally lies in the order of $\sim 20 \%$ from a two-dimensional SAXS analysis of high oriented polymers ${ }^{20-22}$. The product, $\left(l_{a}+l_{c}\right) \delta b_{Z}$, is therefore in the order of 0.4 . From electron microscopy ${ }^{12,13}$ the number of lamellae in one bundle is generally found to be larger than 10 . Therefore $\delta b_{Z} \gg \delta b_{s}$ and in all these cases equation (8b) should hold.

Now, to obtain the peak intensity $\iint I \mathrm{~d} b_{2} \mathrm{~d} b_{3}$ of the bundle one must subtract the background of the SAXS. Part of the background is given by the first term of equation (1). The rest comes from $Z_{3}(b)$ and is given by:

$$
Z_{3 \min } \cong \frac{1-\left|F_{3}\left(b_{3 m}\right)\right|}{1+\left|F_{3}\left(b_{3 m}\right)\right|}
$$

The difference between equations (5) and (9) then gives the contribution of $Z_{3}$ to the peak intensity of the bundle:

$$
Z_{3 p}=\frac{4\left|F_{3}\left(b_{3 m}\right)\right|}{1-\left|F_{3}\left(b_{3 m}\right)\right|^{2}}
$$

The peak intensity of the first meridian reflection of an oriented sample in a microdensitometer with a slit parallel to the equator is therefore given by equation (4). If one introduces equations ( $8 \mathrm{~b}$ ) and (12) the result is:

$$
\bar{I}_{p}=(\Delta \rho)^{2} \frac{\pi D^{2}}{4}\left(\frac{\sin \pi l_{c} b_{3 m}}{\pi b_{3 m}}\right)^{2} N Z_{3 p}
$$

Its position is approximately given in most cases by $h=$ 0.9 since the peak of $Z_{3}\left(b_{3}\right)$ at $h=1$ is multiplied with $\sin \pi l_{c} b_{3 m} / \pi b_{3 m}$, which in most cases decreases drastically with increasing $b_{3}$ in the region of this peak ${ }^{22} .\left|F_{3}\left(b_{3 m}\right)\right|$ is, according to the above mentioned value of $\mathrm{g}(\sim 20 \%)$, in 


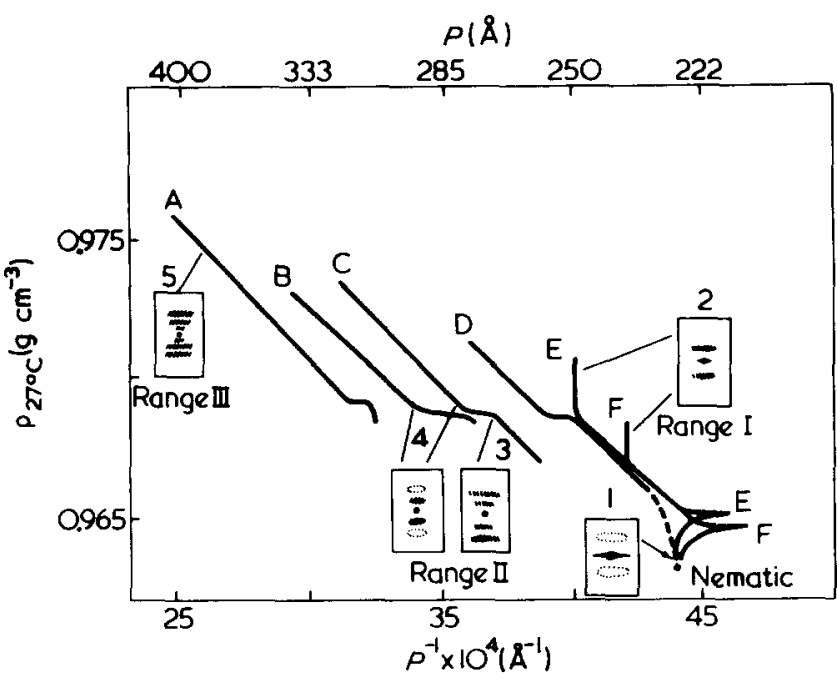

Figure 6 Density, $\rho$, of hot-drawn polyethylene (linear polyethylene LUPOLEN $6001 \mathrm{H}$ stretched) plotted against the reciprocal of long period for various annealing temperatures $\left({ }^{\circ} \mathrm{C}\right): \mathrm{A}, 129 ; \mathrm{B}$. $126 ; C, 120 ; D, 110 ; E, 100 ; F, 95$ and times $^{27}$ in the three temperature ranges and the respective SAXS pattern. $P$ (long period) reaches a finite value after some days according to Figure $3 b$ and grows unbounded in range 111 according to Figure $3 f$. Stretching temperature $T_{1}, 80^{\circ} \mathrm{C}$, draw ratio $9.6 x$

the order of $1 / 2$, hence $4 F_{3}\left(b_{3 m}\right) / 1-\left|F_{3}\left(b_{3 m}\right)\right|^{2}$ is in the order of 3.

If $V$ is the irradiated volume of the sample, $M$ the number of bundles and $\gamma$ the volume fraction of bundles, then the volume occupied by the bundles is given by:

$$
\gamma V=M N\left(l_{a}+l_{c}\right) \pi D^{2} / 4
$$

Introducing this in equation (13) we finally obtain for the $S A X S$ peak intensity:

$$
\overline{I_{p}}=(\Delta \rho)^{2} \gamma V\left(\frac{\sin \pi l_{c} b_{3 m}}{\pi b_{3 m}}\right)^{2} \frac{l_{c}+l_{a}}{\pi} Z_{3 p}
$$

Equation (15) shows that $\bar{I}_{p}$ is proportional to the square of the electron density difference (as seen in annealing range I), to the volume fraction of bundles (as seen in range III) and to $Z_{3 p}$ as defined by equation (12), or in the order of 3 . At a given long period $\left(l_{a}+l_{c}\right)$ it has a maximum for $l_{a}=l_{c}$, which is well-known. The next interesting result is the proportionality to $\left(l_{a}+l_{c}\right)$. Even if the volume fraction $\gamma, l_{a} / l_{c}, g$ and $\Delta \rho$ are constant, $\bar{I}_{p}$ increases if the thickness of the lamellae $\left(l_{a}+l_{c}\right)$ increases uniformly as in annealing range II.

\section{APPLICATION TO ANNEALING EFFECTS OBSERVED IN DRAWN SYSTEMS}

In the case of cold or hot drawn systems, the lamellar morphology is often replaced by a typical fibrillar morphology at sufficiently high extensions (Figure $3 a$ ). When the highly drawn fibrillar systems is annealed in the different temperature ranges I, II and III and studied by means of electron microscopy (e.m.) at room temperatures ${ }^{26}$, results suggest little or no morphological changes taking place at the annealing temperature in range $I$, but that a transformation from a fibrillar to a lamellar morphology must have taken place starting at some temperature in annealing tem. perature range I (Figure $3 b$ ). The generally accepted ex- planation is that at high enough temperature additional molecular mobility allows the lateral alignment of crystallites in neighbouring fibrils as well as the additional growth of lamellar thickness by means of a solid state refolding process.

However, additional information can be obtained about the transformation process from two-dimensional analysis of SAXS patterns obtained at room temperatures of such systems after annealing at various temperatures for various times. Since the details of the SAXS studies have already been reported elsewhere ${ }^{27}$, it is our purpose here to show how the annealing mechanisms described earlier can also be of direct application to the understanding of the annealing effects detected in such non-equilibrated drawn systems that are often encountered during normal fibre or film processings or any future applications of solid state forming processes.

Figure 6 shows a plot of density vs. the inverse of SAXS long period for an annealed hot drawn polyethylene. Both the density and the long period were measured at room temperature after quenching from the various annealing temperatures at the end of various annealing times. There are various interesting characteristics to the curves. When annealed to temperatures below $110^{\circ} \mathrm{C}$ (which we shall refer to as range $I^{\prime}$ ), the observed changes are quite different from those seen after annealing between $\sim 110^{\circ} \mathrm{C}$ and $\sim 130^{\circ} \mathrm{C}$ (range $\mathrm{II}^{\prime}$ ). In range $\mathrm{I}$, after $10^{2} \mathrm{~min}$, the SAXS long period stays nearly constant while $\rho$ increases and finally reaches a limiting value. The voids originally present between the microfibrils as a result of the stretching process are now partially removed after the annealing step, which contributes to the observed increase in density to a limiting value. The limited mobility of the chain segments in range I (Figure 1a) explains the limited increase of long period as well as the lack of lateral alignment of $M P C$ in neighbouring fibrils, from information provided by the SAXS patterns, which showed an $m P C$ packing to be nearly the same as the one depicted in Figure $3 b$.

In range $\mathbf{I I}^{\prime}$, both the long period and density increase with time except for a small plateau, where density stays nearly constant and only long period increases. The extent of this plateau becomes shorter, but it takes place earlier with increasing annealing temperature. Two-dimensional analysis of the SAXS patterns indicates that in temperature range $\mathrm{II}^{\prime}$, the morphology begins to change with annealing time going from a fibrillar to a lamellar structure. This is in complete agreement with similar electron microscopy studies ${ }^{26}$, which not only showed the transformation but that it takes less and less time with increasing temperature after annealing between $110^{\circ}$ and $130^{\circ} \mathrm{C}$. The results from density measurements and SAXS and e.m. studies indicate that the fibrillar-to-lamellar morphological transformation can take place by a similar melting of the exposed small $m P C$ (Figures $3 a, 3 c$ and $3 d$ ), and recrystallization process described earlier. However, because the recrystallization on crystallization from an oriented melt occurs at a much faster rate than recrystallization from an unoriented melt, the solidification process is most likely to have been completed within the annealing times or during quenching to room temperatures after annealing. This explains the differences seen in density changes with time for unoriented and oriented systems; the density for oriented systems generally increases with time whereas for unoriented systems it may show a drop initially before it proceeds to increase with time ${ }^{5}$. We suggest that if the density for our annealed oriented systems is measured fast enough at the 
annealing temperature, we should be able to detect a decrease due to initial melting.

\section{ACKNOWLEDGEMENTS}

The authors gratefully acknowledge the generous support of this work by the Alexander von Humboldt-Stiftung and the Deutsche Forschungsgemeinschaft, Bonn. One of the authors (G. S. Y. Y.) was a recipient of a Senior US Scientist Award of the Alexander von Humboldt-Stiftung and wishs to give special thanks to the Foundation and his host (R. H.) for their generous hospitality.

\section{REFERENCES}

Fischer, E. W. Kolloid-Z. Z. Polym. 1969, 231, 458

Fischer, E. W. Pure Appl. Chem. 1972, 31, 113

Schultz, J. M. 'Polymer Materials Science' Prentice-Hall, New Jersey, 1974

4 Hoffman, J. D. see Fischer, E. W. Kolloid-Z. Z. Polym. 1969, 231, 458; Kilian, H. G., Kolloid-Z. Z. Polym. 1969, 231, 534

5 Fischer, E. W. and Schmidt, G. F. Angew. Chem. 1962, 1, 488

6 Geil, P. H. 'Polymer Single Crystals', Interscience, New York, 1963

7 Luch, D. and Yeh, G. S. Y. J. Macromol. Sci. (B) 1973, 7 , 121
8 Nukushina, Y., Itoh, Y. and Fischer, E. W. J. Polym. Sci. (B) $1965,3,383$

9 Schultz, J. M., Robinson, W. H. and Pound, G. M. J. Polym. Sci. (A) $1967,5,511$

10 Hosemann, R., Wilke, W. and Baltá-Calleja, F. H. Acto Crystallogr. 1966, 21, 118

11 Yeh, G. S. Y. and Geil, P. H. J. Macromol. Sci. (B) 1967, 1, 235, 251; Yeh, G. S. Y. and Lambert, S. L. J. Appl. Phys. 1971, 42, 4614, J. Macromol. Sci. (B) 1972, 6, 599

12 Luch, D. and Yeh, G. S. Y. J. Appl. Phys. 1972, 43, 4326

13 Hardin, I. R. and Yeh, G. S. Y. J. Macromol. Sci. (B). 1973. 7,375

14 Takayanagi, M. J. Macromol. Sci. (B) 1974, 9, 391

15 Strobl, S., Ewen, B., Fischer, E. W. and Piesezek, W. J. Chem. Phys. 1974,61,5257

16 Yeh, G.S. Y. J. Macromol. Sci. (B), 1973, 8, 241

17 Fatou, J. G. and Mandelkern, L. J. Phys. Chem. 1965, 69 , 417

18 Illers, K. H. and Hendus, H. Makromol. Chem. 1968, 113, 1 Hosemann, R. Polymer 1962, 3, 349

Hosemann, R. Ber. Bunsenges. Phys. Chem. 1970, 74, 755 Ferracini, E., Ferrero, A., Loboda-Cačković, J., Hosemann, R. and Cačkovic, H. J. Macromol. Sci. (B) 1974, 10, 97

22 Loboda-Čačković, J., Hosemann, R. and Cačković, H. Kolloid-Z. Z. Polym. 1971, 247, 824

Hermans, J. J. Recl. Trav. Chim. Pays. Bas. 1944, 63, 5 Tsvankin, D. J. Vysokomol. Soedin (A) 1967, 9, 2628 Hosemann, R. and Bagchi, S. N. 'Direct Analysis of Diffraction by Matter', North-Holland, Amsterdam, 1962

26 Yeh, G. S. Y. and Geil, P. H. J. Mater. Sci. 1967, 2, 457

27 Loboda-Cacković, J., Hosemann, R. and Wilke, W. Kolloid-Z. Z. Polym. 1969, 235, 1162 